\title{
Filter Design and Optimization of Electro-Mechanical Actuation Systems Using Search and Surrogate Algorithms for More-Electric Aircraft Applications
}

Gao, Yuan; Yang, Tao; Bozhko, Serhiy; Wheeler, Patrick; Dragicevic, Tomislav

Published in:

IEEE Transactions on Transportation Electrification

Link to article, DOI:

10.1109/TTE.2020.3019729

Publication date:

2020

Document Version

Peer reviewed version

Link back to DTU Orbit

Citation (APA):

Gao, Y., Yang, T., Bozhko, S., Wheeler, P., \& Dragicevic, T. (2020). Filter Design and Optimization of ElectroMechanical Actuation Systems Using Search and Surrogate Algorithms for More-Electric Aircraft Applications. IEEE Transactions on Transportation Electrification, 6(4), 1434 - 1447. https://doi.org/10.1109/TTE.2020.3019729

\section{General rights}

Copyright and moral rights for the publications made accessible in the public portal are retained by the authors and/or other copyright owners and it is a condition of accessing publications that users recognise and abide by the legal requirements associated with these rights.

- Users may download and print one copy of any publication from the public portal for the purpose of private study or research.

- You may not further distribute the material or use it for any profit-making activity or commercial gain

- You may freely distribute the URL identifying the publication in the public portal 


\title{
Filter Design and Optimization of Electro-Mechanical Actuation Systems Using Search and Surrogate Algorithms for More-Electric Aircraft Applications
}

\author{
Yuan Gao, Student Member, IEEE, Tao Yang, Senior Member, IEEE, Serhiy Bozhko, Senior Member, IEEE, \\ Patrick Wheeler, Senior Member, IEEE and Tomislav Dragičević, Senior Member, IEEE
}

\begin{abstract}
In this paper, a de filter design and optimization problem is studied for dc electrical power distribution systems onboard more-electric aircraft. Component sizing models are built to serve as the basis of the optimization whose objectives are mass and power loss of this filter. A categorization strategy of search and surrogate algorithms are proposed and used for the target multi-objective optimization problem (MOOP). A genetic algorithm is utilized as a search algorithm to identify potential best solutions based on a set of filter sizing functions (subject to constraints). Additionally, two machine learning (ML) algorithms are considered as surrogate algorithms to address the same optimization problem. In the ML training process, a constraint violation model is applied since there are various constraints in optimization and this kind of classification model is relatively difficult to train. A support vector machine is applied for the constraint violation model after which two artificial neural networks are trained as the final surrogate model for mapping design variables to filter performance. To address these issues, a novel category of search and surrogate algorithms are proposed. Both algorithms are explored to solve the filter MOOP and their optimization results are compared at the end.
\end{abstract}

Index Terms-Filter design, optimization, genetic algorithm (GA), search algorithm, surrogate algorithm, artificial neural network (ANN), support vector machine (SVM), more-electric aircraft (MEA)

\section{NOMENCLATURE}

$x_{1}, x_{2} \ldots \quad$ Inputs of algorithms.

$o_{1}, o_{2} \ldots \quad$ Outputs of algorithms.

$H, H_{\max } \quad$ Magnetic field strength inductor core.

A Magnetic enclosed path in inductor.

$I_{L}, I_{L, \max } \mathrm{RMS}$ and peak value of inductor current.

$J, J_{\max } \quad$ Current density and max value in inductor coil.

Manuscript received February 28, 2020; first revised June 2, 2020; second revised July 20, 2020; accepted August 22, 2020. This work was supported by the Clean Sky 2 Joint Undertaking under the European Union's Horizon 2020 research and innovation programme under grant agreement No 807081. (Corresponding Author: Tao Yang).

Y. Gao, T. Yang, S. Bozhko and P. Wheeler are with the Department of Electrical and Electronic Engineering, University of Nottingham, Nottingham, UK (e-mail: yuan.gao@nottingham.ac.uk; tao.yang@nottingham.ac.uk; serhiy.bozhko@nottingham.ac.uk; pat.wheeler@nottingham.ac.uk).

T. Dragicevic is with the Department of Energy Technology, Technical University of Denmark, Lyngby, Denmark (email: tomdr@elektro.dtu.dk).

\begin{tabular}{|c|c|}
\hline$d_{w}$ & Wire diameter of inductor winding. \\
\hline$h_{\text {ins }}$ & Insulation thickness of inductor wire. \\
\hline$N_{t}, N_{t, \max }$ & Number of turns and its max value. \\
\hline$C_{\text {window }}$ & Circumference of inner cycle of inductor core. \\
\hline$A_{c, \min }$ & Min core cross-section area. \\
\hline$B_{\max }$ & Max flux density in inductor core. \\
\hline$\mu$ & Magnetic permeability. \\
\hline$\gamma, \gamma_{\max }$ & Magnetic permeability drop and its max value. \\
\hline$C_{1}$ & $L C$ filter capacitance. \\
\hline$R_{1}, R_{2}$ & Internal resistance of inductor and capacitor \\
\hline & Nominal inductance factor of inductor core. \\
\hline$P L_{L}, P L_{C}$ & Inductor power loss and capacitor power loss. \\
\hline$T_{\text {rise }}, T_{\text {lim }}$ & Inductor temperature rise and its limitation. \\
\hline$A_{c, s}$ & Inductor core surface area. \\
\hline ESR & Equivalent series resistance of capacitor. \\
\hline $\tan \delta$ & Dissipation factor of capacitor series. \\
\hline$R_{t h}$ & Thermal resistance of capacitor. \\
\hline$I_{C}, I_{\max }^{R M S}$ & RMS current of capacitor and its limit value. \\
\hline$T_{a m b}$ & Ambient temperature for capacitor. \\
\hline$v_{i}$ & $\begin{array}{l}\text { Possible voltage vectors across ac loads }(i= \\
1,2 \cdots 7) \text {. }\end{array}$ \\
\hline$v_{n N}$ & Common mode voltage drop. \\
\hline$v_{y N}$ & Voltages across the 3-phase loads $(y=a, b, c)$. \\
\hline$e_{0}$ & Motor \\
\hline$R_{m}$ & AC resistance. \\
\hline$L_{m}$ & Motor inductance. \\
\hline$i_{d c}$ & DC inductor current. \\
\hline$i_{o}, i_{o}^{*}$ & $\mathrm{AC}$ current and its control reference. \\
\hline$i_{\max }$ & AC current limitation. \\
\hline$L_{0}, R_{0}$ & DC microgrid equivalent impedance. \\
\hline$i_{p o l, i}, i_{p o l, f}$ & $\begin{array}{l}\text { DC initial and final currents flowing into con- } \\
\text { verter during a time step. }\end{array}$ \\
\hline$v_{s}, v_{d c}$ & Pure source voltage, dc supply voltage. \\
\hline$v_{C}, v_{C}^{*}$ & DC capacitor voltage and its control reference. \\
\hline & Weighting factor of DC voltage control term. \\
\hline$g_{\text {con }}, g_{C}$ & $\begin{array}{l}\text { AC current and DC voltage control terms in } \\
\text { cost function of FCS-MPC. }\end{array}$ \\
\hline $\begin{array}{l}g_{t}, G_{l i m} \\
M, M_{\max }\end{array}$ & Total cost function and its current limit term. \\
\hline & \\
\hline$P L, P L_{\max }$ & Total power loss of $L C$ filter. \\
\hline & \\
\hline
\end{tabular}

$d_{w}$

$C_{\text {window }}$

$A_{c, \min }$

$B_{\max }$

$\gamma, \gamma_{\max }$

$L_{1}$

C

$R_{1}, R_{2}$

$P L_{L}, P L_{C}$ Inductor power loss and capacitor power loss

$T_{\text {rise }}, T_{\text {lim }}$

ESR

$\tan \delta$

$I_{C}, I_{\max }^{R M S}$

$T_{a m b}$

$v_{i}$

$v_{n N}$

$v_{y N}$

$e_{0}$

$i_{d c}$

$i_{o}, i_{o}^{*}$

$i_{\max }$

$L_{0}, R_{0}$

$v_{s}, v_{d c}$

$v_{C}, v_{C}^{*}$

$\lambda_{d c}$

$S_{a}, S_{b}, S_{c}$

Number of turns and its max value.

Min core cross-section area.

Max flux density in inductor core.

Magnetic permeability.

$L C$ filter inductance.

$L C$ filter capacitance.

Inductor core surface area

Equivalent series resistance of capacitor

Thermal resistance of capacitor.

Ambient temperature for capacitor.

$1,2 \cdots 7)$

Common mode voltage drop.

Motor back-EMF.

AC resistance.

Motor inductance.

AC current limitation.

Wire diameter of inductor winding.

. (n) 


$$
\begin{array}{ll}
x_{a}, x_{b}, x_{c} & \text { Three-phase voltage variables. } \\
T_{s} & \text { Sampling period in FCS-MPC. } \\
r & \text { Integrated index of two optimization objec- } \\
& \text { tives. }
\end{array}
$$

\section{INTRODUCTION}

W ITH the ongoing continuous development of the moreelectric aircraft (MEA) [1]-[7] concept, replacing the ac electrical power systems (EPS) with a dc distribution systems has become a new research direction and development tendency [1], [2], [8], [9]. Compared with ac systems, dc systems are much simpler to control and they have no reactive power thus don't need compensation devices. This will save cable weight and improve efficiency of electrical energy use in MEA [1], [2], [6]. In a dc EPS, the power quality on the $\mathrm{dc}$ bus is of great concern as there could be distortions due to the operation of power electronic connections. In order to provide a good quality power supply to a power-converterfed Electro-Mechanical Actuator (EMA) [1], [3], [4], [7], this paper studies an $L C$ filter design for a point of load (POL) actuator. To achieve the design and optimization of this $L C$ filter, two kinds of algorithms are used and compared.

The conventional power distribution in aircraft utilizes a single-generator-per-bus paradigm integrated with switching components [1], [2]. In [2], a 'single-bus' concept was proposed for an on-board dc micro-grid (MG) where all sources and loads are connected to a single distribution dc bus (Fig. 1). The motivation for adding a filter feeding POL actuator is from the potential voltage destabilization on the dc bus. In the MEA concept, the main generator is directly coupled to the jet engine thus the frequency of generator output power bus is proportional to engine speed [7]. In a generalized dc EPS, the power supply could have many frequency components in different flight phases (for example take-off, cruise, descent, landing and taxiing) due to the variations in engine speed. Besides, the dc bus may have a large ripple due to the actively power control devices connected to control the bus voltage. This underlies the interest of applying a filter to the potential aircraft EMAs.

Filter design methods commonly incorporate sizing models for inductor and capacitor where physical parameters (for example weight, size etc.), inner resistance and thermal performance are considered [10]-[13]. An optimization approach for the passive components for rectifiers is presented in [10], but it only considers a one boost inductor topology [11] using commercial cores. In [13] an $L C L$ harmonic filter is designed based on a generic optimization approach to minimize losses, but the damping branch design is not clearly described. A comprehensive non-iterative analytical $L C L$ filter design method is presented in [12] where the converter current ripple was analyzed to determine the limit of boost inductance. Different from these papers, the inductor is used in a dc line thus the core loss [14] of inductor is not considered here. Furthermore, toroidal powder cores are selected for the inductor design thus no air gap is considered in inductor. The capacitor is designed using a similar method in which dissipation factors enable capacitor loss estimation [10], [13]. The $L C$ values for

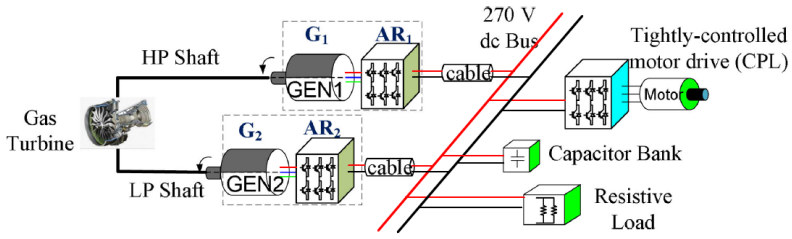

Fig. 1. One possible EPS in the MEA [2].

the filter are determined conventionally using current ripples or required harmonic attenuation [10]-[13], [15]; whilst this study applies a reverse thought and directly treats $L C$ as design variables (DVs). In this paper the current ripple is carefully considered in the constraints of optimization and desired passive values can be obtained directly from the operation of search algorithm. Lastly, the internal filter resistance and its damping effect [15] are considered in the automated integrated sizing process.

Another new contribution included in this paper is the integrated application of two kinds of algorithms, the search algorithm [16], [17] and the surrogate algorithm [18]-[21], for $L C$ filter optimization. The category strategy of the two algorithms is proposed and their relationships are discussed. Then, the target $L C$ filter optimization problem (MOOP) is studied based on an established inverter-based EMA system [4] using a search algorithm, genetic algorithm (GA) [16], [17], [22], which is similar to the algorithm used in [3]. Furthermore, machine learning (ML) algorithms are explored as the surrogate algorithms for the same MOOP and there are two data-driven modes: classification [20], [21] and fitting [18], [19], [23], [24]. This paper uses both modes to demonstrate the surrogate algorithm and proposes a ML structure for the target MOOP which can be generally used in any discipline. This structure is divided into two parts: constraint violation model (using a classification mode) and performance mapping model (using a fitting mode). After building these two surrogate models, the filter optimization results from search and surrogate algorithms are compared. The motivation is mainly due to the surrogate algorithms representing some advantages over search algorithms. Once trained a filter surrogate model, it can be used directly in any future study of this MOOP instead of running the detailed EMA system model with search algorithm over and over again, which can be very time-consuming.

This paper is organised as follows: In Section II, the category of the studied algorithms is proposed, which serves as the basis of this paper; Sections III \& IV discuss filter sizing models and the control strategy for a converter fed EMA; After that, the procedure of GA (search algorithm) for the optimal filter design is introduced in Section V. In Section VI, two ML algorithms (surrogate algorithm) are developed and used for the same MOOP design. Optimization results from different optimization methods (GA, ML algorithms) are compared in Section VI.

\section{Search Algorithm And Surrogate Algorithm} (2SA)

In this section, we give a novel category strategy for different commonly used algorithms including GA, particle swarm 


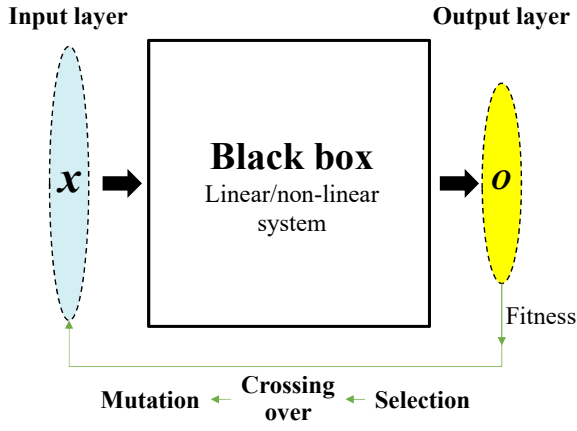

(a) Search algorithm.

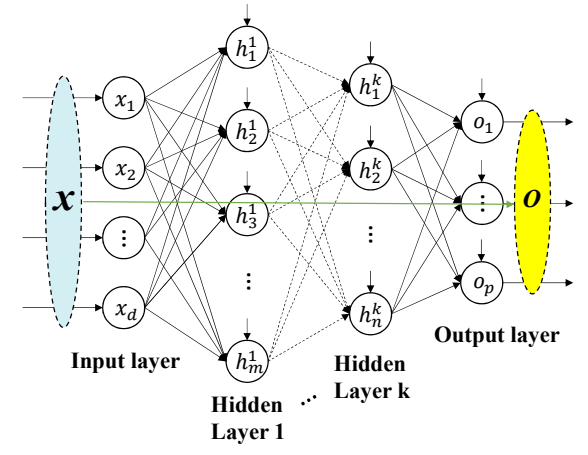

(b) Surrogate algorithm.

Fig. 2. The proposed "2SA" categorization (giving GA and ANN as the examples).

[25], tabu search [16], [26] and artificial neural network (ANN) [18], [19], [23], [24], Bayesian network [27], support vector machine (SVM) [20] etc. Based on this strategy, the relations $\left(\mathbf{x}\right.$-to-o) between inputs $\left(x_{1}, x_{2} \ldots\right)$ and outputs $\left(o_{1}, o_{2} \ldots\right)$ in algorithm categories can be simply described and compared. As shown in Fig. 2, there are two categories: search algorithm and surrogate algorithm, abbreviated as ' $2 \mathrm{SA}$ '. Both are describing the relations of $\mathbf{x}$-to-o but their logic directions (green arrows in Fig. 2) are inverse: for search algorithm the direction is from outputs to inputs which is 'outside' of a studied system; in contrast, the logic direction of surrogate is 'inside' the system because data in the algorithm operation is always flowing from inputs to outputs (x-to-o). However, the former logic direction is not a data-flow direction but a 'command', it means data in $\left(o_{1}, o_{2} \ldots\right)$ can change $\left(x_{1}, x_{2} \ldots\right)$ in certain ways which are specific in different search algorithms (e.g. selection, cross over, mutation in GA). Though, in surrogate algorithms, there is a 'command' that $\left(o_{1}, o_{2} \ldots\right)$ can change the training parameters in surrogate algorithms (e.g. weights, bias in ANN), $\left(x_{1}, x_{2} \ldots\right)$ in surrogate algorithm is usually not changed by $\left(o_{1}, o_{2} \ldots\right)$.

\section{A. Search Algorithm}

The search algorithm treats a studied system as a black box (no matter linear or non-linear). The search algorithm is to calculate the fitness of each design based on $\left(o_{1}, o_{2} \ldots\right)$ (current generation) and then determine new $\left(x_{1}, x_{2} \ldots\right)$ in the next generation according to specific algorithm theories. As will be discussed in Section V.A, GA searching process relies on bioinspired operators such as selection, crossover and mutation [16], [17]. Other algorithms or variants such as particle swarm [25], tabu search [16], [26], NSGA II [28] and NSGA III [29], [30] also fit this characteristic thus can be categorized as search algorithms.

\section{B. Surrogate Algorithm}

The other category in 2SA, surrogate algorithm, is to build a math mapping model based on a set of samples. The mapping model can make predictions effectively without running the original system(s) being explicitly programmed. In training, internal algorithm parameters are continually updated by using the errors between samples (raw data) and current predictions [18], [19]. Therefore, a mapping/surrogate model is established by a supervised learning until when it can be used to replace the original system(s) to perform a task. That is the reason why it has been named "surrogat", i.e. to reflect the replacement function.

Fig. 2(b) is a schematic of a forward ANN (weights and biases are omitted for simplicity). The number of neurons in $\left(x_{1}, x_{2} \ldots\right)$ and $\left(o_{1}, o_{2} \ldots\right)$ layers are determined by the samples. The neuron number in hidden layers can be set an ANN algorithm developer. Therefore, neuron number selection for different ANN structures is flexible and this has been a persistent and hot topic in multi-discipline studies [31][33]. Bayesian network, SVM and other ML methods can also be trained and used in similar ways thus they should be categorised as surrogate algorithms. Besides, as discussed, the surrogate models can be further divided into two modes as surrogate fitting and surrogate classification whose output types are different. A case of two modes will be given in Section VI.

It is important to note that the surrogate algorithms described here are offline algorithms which do not consider system uncertainty, variations in different time steps or noise models in training. Some online ML algorithms which store past experience and compute optimal policies that maximize the long-term reward are out of the scope (for example Deep Deterministic Policy Gradient [34]).

\section{Comparison between search algorithm and surrogate al- gorithm}

Differences and connections of 2SA are concluded in Table I. Search algorithm is to do the optimization while surrogate algorithm is used to train a data-based 'agent' mapping $\mathbf{x}$ to-o relations but cannot do optimization itself. However, surrogate algorithm can be trained as an agent to effectively serve optimization [18], [19], [35]. Besides, the operation of search algorithm is a data changing process (both inputs and outputs) but for a surrogate model, these data usually stay unchanged in training. Last but not the least, in order to optimize the system using surrogate algorithms, an exhaustive method can be applied based on the trained surrogate which gives predictions quickly [19].

Based on the developed concepts, this paper will use a GA tool and then establish a generic surrogate structure to 
TABLE I

Differences And Connections Between SEARCh Algorithm AND Surrogate Algorithm

\begin{tabular}{lll}
\hline \hline & Search algorithm & Surrogate algorithm \\
\hline Purpose & Used for the optimization in a certain design space. & $\begin{array}{l}\text { Used to train a data-based 'agent' which maps specific } \\
\text { relations but cannot do optimization by itself. }\end{array}$ \\
Operation mechanism & $\begin{array}{l}\text { Calculate the 'fitness' of outputs and determine new } \\
\text { inputs according to the algorithm theory. }\end{array}$ & $\begin{array}{l}\text { Based on raw data, build a mathematical 'agent' model } \\
\text { by updating algorithm parameters. }\end{array}$ \\
Data in operation & $\begin{array}{l}\text { Both input and output data are changing during opti- } \\
\text { mization process. }\end{array}$ & $\begin{array}{l}\text { Raw or sample data usually stays unchanged and the } \\
\text { model is trained offline. }\end{array}$ \\
Model building & $\begin{array}{l}\text { Can use the trained 'agent' to help find the search } \\
\text { boundaries for search algorithm operation. }\end{array}$ & $\begin{array}{l}\text { Can use search algorithm to find the optimum param- } \\
\text { Interactive functions a desired surrogate model. }\end{array}$ \\
$\begin{array}{l}\text { Can utilize the trained 'agent' model to generate } \\
\text { outputs for the corresponding inputs to serve the } \\
\text { optimization. }\end{array}$ & $\begin{array}{l}\text { After training, search algorithms can help do the } \\
\text { following optimization works in a certain design space. }\end{array}$ \\
\hline \hline
\end{tabular}

optimize the $L C$ filter for EMA. However, before we discuss the operation of $2 \mathrm{SA}$ models, we may have to introduce $L C$ filter sizing models and system control strategy in the following two sections.

\section{FILTER Sizing MOdelS}

The $L C$ values of filter are calculated using current ripple/THD limits or required harmonic attenuation in [10], [13], [15]. However, these equations can only give extreme values of $L C$ which do not guarantee the minimum mass and loss of the whole filter system. To solve this issue, commercial data based filter optimization can be used [10], [13]. This paper presents an automated and integrated optimal filter design method with the embedded circuit simulation where all the required ripple/THD are carefully considered as the optimization constraints. This section will present the sizing models with input $L$ and $C$ to serve the optimization. The sizing models provide a method to independently estimate the size of a inductor and a capacitor within an $L C$ filter. However, the developed model can be applied to any types of filters. Both sizing process of inductors and capacitors consider power loss and mass as the performance indices. In this section, the sizing function of inductors and capacitors will be introduced separately.

\section{A. Inductor Sizing Function}

To develop sizing functions for inductors, inductor architectures should be first confirmed. Popular inductor shape and architectures are mainly of two types: discrete-gap cores with laminated ferrite/iron-based alloys, or distributed-gap cores with powdered iron-based alloys. Distributed-gap cores offer the advantage of having soft magnetic saturation characteristics, allowing a gradual drop in inductance as DC-bias current is increased. As a result, distributed-gap cores can be designed to be smaller in size for the same inductance thus, they are more suitable for aerospace applications [11]. Toroidal powder cores made by magnetic material MPP60 from Magnetics Inc. [36] are utilized in this study. This sizing model essentially

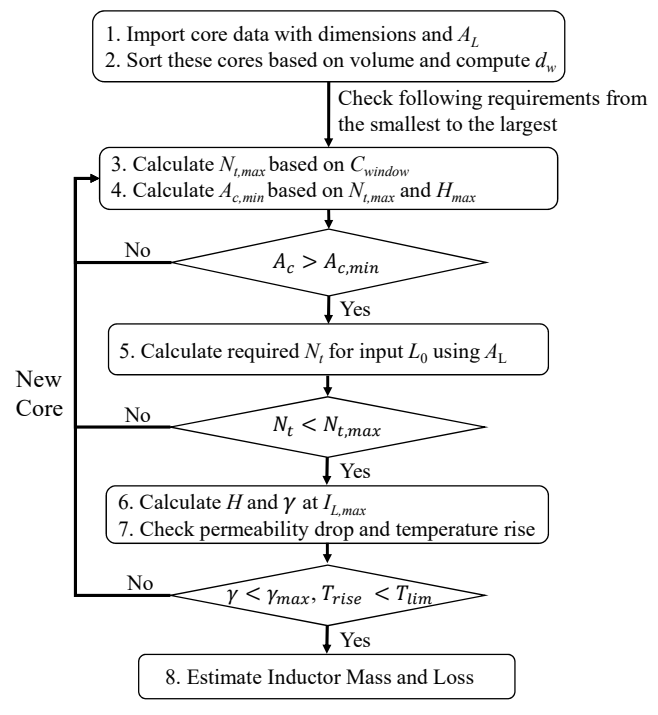

Fig. 3. Inductor sizing function.

utilizes the Maxwell-Ampere's law (1) to calculate conductor cross-sectional area with current RMS value [11]-[13], [36]:

$$
\oint H \cdot d l=\int_{A} J \cdot d A=\sum i(t)
$$

where $H(A / m)$ denotes the magnetic field strength along an enclosed path with length $l(m), J\left(A / m^{2}\right)$ represents the current density of current $i(A)$ flowing through a crosssectional area of $A\left(m^{2}\right)$. As shown in Fig. 3, at the beginning of the sizing inductors procedure, discrete core sizes and data from Magnetics Inc. are imported to a selection pool. And several constraints, e.g. minimum cross-section area $A_{c, \text { min }}$, maximum number of turns $N_{t, \max }$, permeability drop $\gamma_{\max }$ and temperature rise $T_{l i m}$, must be checked one by one before generating feasible cores for the desired $L_{1}$. After that, the smallest core is chosen from the remaining feasible cores, inductor mass and loss can hence be given to the GA operators for optimization.

The RMS current $I_{L}$ and peak current $I_{L, \max }$ flowing into 
the inductor should be given by simulation. The required coil diameter can be computed as [11]:

$$
d_{w}=2 \sqrt{\frac{I_{L}}{\pi J_{\max }}}+2 h_{\text {ins }}
$$

where $J_{\max }$ is the maximum RMS current density [10] and $h_{\text {ins }}$ is the insulation thickness. With this coil diameter, the maximum number of turns $N_{t, \max }$ can be calculated for a core using core's internal diameter and minimum space between conductors, which are limited at the circumference $\left(C_{\text {window }}\right)$ of the inner cycle of each core. Then, the minimum core crosssection area $A_{c, \min }$ can be give by:

$$
A_{c, \text { min }}=\frac{\Theta}{B_{\max }}=\frac{I_{L} L_{1}}{\gamma \mu H_{\max } N_{t, \max }},
$$

where $\mu$ is the magnetic permeability (with its drop $\gamma$ ), $H_{\max }$ is the predefined max field strength [11], [36]. Then, the required number of turns $N_{t}$ with the core's nominal inductance factor $A_{L}$ for $L_{1}$ can be given as :

$$
N_{t}=\sqrt{\frac{L_{1}}{A_{L}}}
$$

In addition, for magnetic constraints, as magnetic force $H$ within the toroidal core increases, permeability $\mu$ drops due to saturation of the material. This relationship between permeability drop $\gamma$ and $H$ is specific to the core material, and it can be found from the material data-sheet. After the resulting $H$ in the core is obtained from $N_{t} * I_{L, \max } / l, \gamma$ of the potential inductor design can be interpolated from the material's $\gamma(H)$ relationship, it should be smaller than $\gamma_{\max }(0.8$ at this work). $R_{1}$ is then obtained by $N_{t}, d_{w}$, copper conductivity and turn length. Regarding the core temperature rise, it can be estimated using (5) [36] and the maximum limitation of rise is set as 55 $\left.{ }^{\circ} \mathrm{C}\right)$ :

$$
T_{\text {rise }}=\left(\frac{P L_{L}}{A_{c, s}}\right)^{0.833}
$$

where $P L_{L}$ is the inductor loss (equals $I_{L}^{2} R_{1}$ ), $A_{c, s}$ is the core surface area.

\section{B. Capacitor Sizing Function}

For DC-link capacitor applications, the two most popular capacitor types are aluminum electrolytic capacitor and polypropylene film capacitor. Although electrolytic capacitors have higher energy storage capacities, film capacitors are known to have better temperature characteristics and current ripples tolerance, due to their lower dielectric loss tangents. Therefore, the capacitors here are selected from DC film capacitors series, more specifically the EPCOS B2562* MKP [37]. The selected series have a rated voltage of $700 \mathrm{~V}$ and the dissipation factor is specified as (at $100 \mathrm{~Hz}$ ):

$$
\tan \delta=\left\{\begin{array}{l}
1.2 \times 10^{-3}, C_{1}<450 \mu \mathrm{F} \\
1.5 \times 10^{-3}, 450 \mu \mathrm{F} \leq C_{1} \leq 800 \mu \mathrm{F} \\
2.0 \times 10^{-3}, C_{1}>800 \mu \mathrm{F}
\end{array}\right.
$$

which enables the Equivalent Series Resistance (ESR) calculation of capacitor and further estimates power loss.

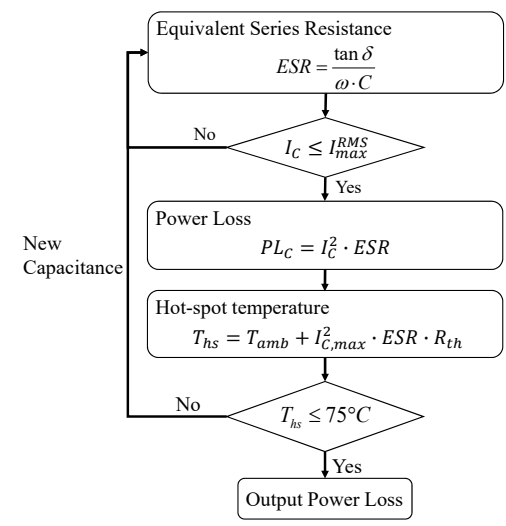

(a) Power loss estimation.

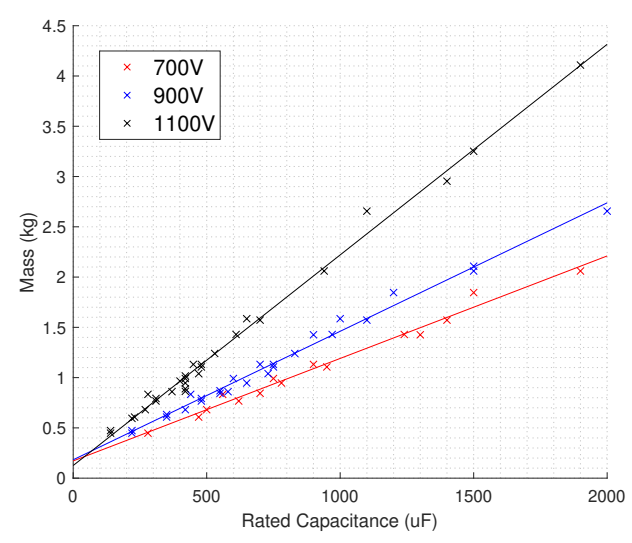

(b) Mass linear fitting for B2562* capacitors.

Fig. 4. Capacitor sizing function.

Fig. 4(a) shows the loss estimation with two constraints of current and temperature. First, the RMS current $I_{C}$ of capacitor should be smaller than the current limitation $I_{m a x}^{\mathrm{RMS}}$ given by manufacture. Then, capacitor loss $P L_{C}$ can be computed using ESR and $I_{C}$. In addition, the thermal model in it consists of only one thermal resistance ( $R_{t h}$, from manufacture) [13], [37] and the ambient temperature $T_{a m b}$ is assumed to be constant at $20{ }^{\circ} \mathrm{C}$. Temperature rise is simply computed by the $\max$ capacitor current $I_{C, \max }, \mathrm{ESR}$ and $R_{t h}$. If the temperature rise is smaller than $55^{\circ} \mathrm{C}$, the capacitor sizing succeeds, its mass and loss can be finally output.

Using a linearly fitting technique in Matlab, the relationship between the capacitance and mass from the component category book [37] can be obtained. Thus, the capacitance density can be approximated to derive the capacitors' mass in the integrated filter sizing. The fitting lines for three capacitor sets (rated voltage $700 \mathrm{~V}, 900 \mathrm{~V}$ and $1100 \mathrm{~V}$ ) in B2562* series are depicted in Fig. 4(b) with the commercial data. The fitting technique gives good linear relationships with fitting $\mathrm{R}^{2}$ results of $0.984,0.989$ and 0.993 respectively.

\section{Integrated Sizing for Filter Optimization}

As discussed in two sizing functions above, currents of inductors and capacitors should be obtained before giving their inner resistance and judgement for constraints. But the 


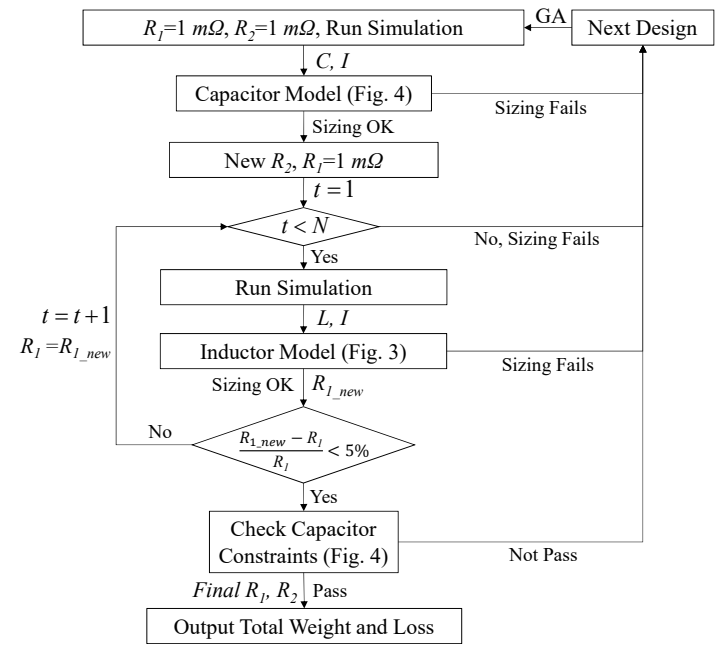

Fig. 5. Integrated sizing for filter optimization using independent inductor and capacitor sizing models.

issue is that the current can only be generated by simulating the system with resistance beforehand input. To solve this problem, an integrated filter design approach is proposed to find the inner resistance with variable values of the passive components given by GA operator, as shown in Fig. 5. $R_{1}$ and $R_{2}$ are both assumed as $1 \mathrm{~m} \Omega$ initially, with that the system can be simulated for each set of DVs. Then, the current signal is processed and given to the capacitor model for the ESR calculation. After that, a simulation iteration is exercised to design the inductor and deduce its inner resistance where the error for iterative resistance is set as 5\% (ESR stays unchanged). Finally, capacitor model is utilized again for the constraint violation checking using data from the last simulation of the iteration, and this is followed by the output of the objective values (total mass and power loss of the desired filter).

\section{Control Strategy in LC Filtered Aircraft ACTUATION SYSTEM}

The circuit diagram of an aircraft actuation system is shown in Fig. 6 where a two-level voltage source converter (VSC) feeding a three-phase motor is supplied from dc bus through an $L C$ filter. On the left, an ideal voltage source $v_{s}$ and a inductor $L_{0}$ with the internal resistance $R_{0}$ are set as a grid supply feeding the studied $L C$ filtered actuator. We apply the finite control set model predictive control (FCS-MPC) [18], [38], [39] to regulate both ac motor current and dc-link voltage in the studied EMA. FCS-MPC is a significant gain over linear control methods where control parameters need to be carefully tuned to ensure stability [40]. The switching of FCS-MPC is not restricted to a predetermined switching pattern of PulseWidth Modulation (PWM) and it allows much better transient response compare to linear control methods [38].

As the control strategy applied to VSC determines the stabilization performance on both ac and dc sides, and at the same time the weighting factor (WF) of FCS-MPC control terms should be well selected, the following subsections will introduce the FCS-MPC based stabilization approach.

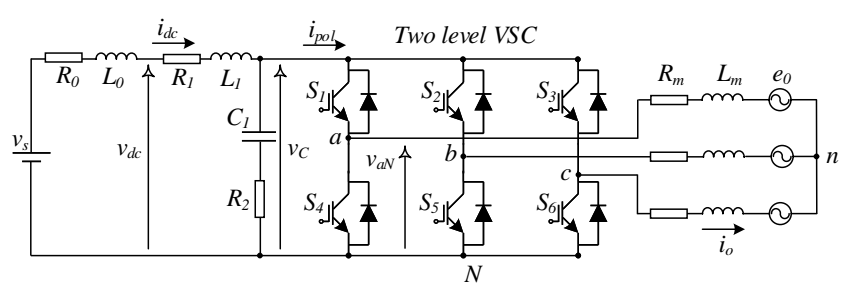

Fig. 6. EMA case study: Two-level VSC fed from a dc grid via LC filter and supplying an ac motor.

TABLE II

Voltage Vectors Used in Two-Level Converter

\begin{tabular}{ccc}
\hline \hline$\left(S_{a}, S_{b}, S_{c}\right)$ & Voltage vector $\bar{v}_{i}$ & $\left(x_{a}, x_{b}, x_{c}\right)$ \\
\hline$(0,0,0)$ & $\bar{v}_{0}=0$ & $(0,0,0) v_{C}$ \\
$(1,0,0)$ & $\bar{v}_{1}=\frac{2}{3} v_{C}$ & $\left(\frac{2}{3},-\frac{1}{3},-\frac{1}{3}\right) v_{C}$ \\
$(1,1,0)$ & $\bar{v}_{2}=\frac{1}{3} v_{C}+j \frac{\sqrt{3}}{3} v_{C}$ & $\left(\frac{1}{3},-\frac{1}{3},-\frac{2}{3}\right) v_{C}$ \\
$(0,1,0)$ & $\bar{v}_{3}=-\frac{1}{3} v_{C}+j \frac{\sqrt{3}}{3} v_{C}$ & $\left(-\frac{1}{3}, \frac{2}{3},-\frac{1}{3}\right) v_{C}$ \\
$(0,1,1)$ & $\bar{v}_{4}=-\frac{2}{3} v_{C}$ & $\left(-\frac{2}{3}, \frac{1}{3}, \frac{1}{3}\right) v_{C}$ \\
$(0,0,1)$ & $\bar{v}_{5}=-\frac{1}{3} v_{C}-j \frac{\sqrt{3}}{3} v_{C}$ & $\left(-\frac{1}{3},-\frac{1}{3}, \frac{2}{3}\right) v_{C}$ \\
$(1,0,1)$ & $\bar{v}_{6}=\frac{1}{3} v_{C}-j \frac{\sqrt{3}}{3} v_{C}$ & $\left(\frac{1}{3},-\frac{2}{3}, \frac{1}{3}\right) v_{C}$ \\
$(1,1,1)$ & $\bar{v}_{7}=0$ & $(0,0,0) v_{C}$ \\
\hline
\end{tabular}

\section{A. FCS-MPC Operating Principle}

In the studied actuator system, FCS-MPC is utilized to regulate both the dc-link voltage $v_{C}$ and the ac motor current $i_{0}$. Regarding the derivation of FCS-MPC, a stationary $\alpha-\beta$ reference frame is applied for VSC modelling. Thus, all the three-phase variables $x_{a}, x_{b}$ and $x_{c}$, are transformed into a corresponding $\alpha-\beta$ frame by applying an amplitude-invariant Clarke transformation [38], [39], [41].

In two-level converter switching functions, $S_{a}, S_{b}, S_{c}$ are the gating signals $(0-1)$. There are totally 8 voltage vectors with 6 active vectors and 2 zero vectors. A common mode voltage drop that results in reduction of voltage across the filter given as:

$$
v_{n N}=\frac{v_{a N}+v_{b N}+v_{c N}}{3} .
$$

The voltages across the ac motor can thus be given as: $v_{a n}=v_{a N}-v_{n N}=S_{a} \cdot v_{C}-v_{n N}, v_{b n}=v_{b N}-v_{n N}=$ $S_{b} \cdot v_{C}-v_{n N}$ and $v_{c n}=v_{c N}-v_{n N}=S_{c} \cdot v_{C}-v_{n N}$. With $v_{a n}, v_{b n}$ and $v_{c n}$, the 8 possible voltage combinations of the inductor voltage on load side can be given by using the Clarke transformation. These voltage vectors, represented as $\bar{v}_{i}=v_{i \alpha}+j v_{i \beta}$, can be found in Table II. Different from the operation of FCS-MPC in [18], [38], this study deals with an ac motor rather than linear loads thus FCS-MPC regulates the ac current instead of the ac capacitor voltage. The 8 possible voltage vectors can be applied to ac resistance $R_{m}$ (with a long cable), motor inductance $L_{m}$ and back-EMF $e_{0}$. The equation that describes the dynamics of the motor current based on the voltage vector $v_{i}$ is given as [39]:

$$
L_{m} \frac{d \bar{i}_{o}}{d t}=\bar{v}_{i}-R_{m} \bar{i}_{o}-\bar{e}_{0}
$$

Apart from relations that describe the motor side, dynamics on the dc side, as shown in the Fig. 7, should also be 
considered. The inductance $L_{0}$ in series with the resistance $R_{0}$ represent the internal dc-network impedance, and the $L C$ filter accounts for inner resistances $R_{1}, R_{2}$ for the inductance $L_{1}$ and the capacitance $C_{1}$ respectively. Only a differential equation describing $v_{C}$ is used for control modelling of the dc capacitor dynamics where the current $i_{d c}$, which flows through $L_{1}$, is treated as an external disturbance:

$$
C_{1} \frac{d v_{C}}{d t}=i_{d c}-i_{p o l}
$$

where $i_{p o l}$ is the current flowing into the VSC. It can be synthesized from the load currents and the gating signals, as:

$$
i_{p o l}=S_{a} i_{f a}+S_{b} i_{f b}+S_{c} i_{f c} .
$$

The equations above define the continuous state-space model of a two-level three-phase VSC with dynamics on both ac and dc sides. The zero-order hold $(\mathrm{ZOH})$ discretization method is used for the predictive control model which can ensure the consistency of discrete time and continuous time at the sampling instants. The simulation system was built in Matlab/Simulink where a predictive controller makes contribution to giving the gating signals to be applied at the next sampling instant. To this end, the back-EMF $e_{0}$ estimation of the load and the prediction of ac current $i_{o}$ and capacitor voltage $v_{C}$ should be done in a sampling period. The backEMF estimation (at $k$ instant) is using the transformation of (8) while this equation also predicts the $k+1$ current using all 8 voltage vectors $v_{i}$ to give candidate value for CF. Then, the capacitor voltage can be estimated by the state of charge (SOC) of this capacitor during one sampling period using an averaging operation [38], [41]:

$v_{C}(k+1)=v_{C}(k)+\left(i_{d c}-\frac{i_{p o l, i}+i_{p o l, f}}{2}\right) \cdot\left(\frac{T_{s}}{C_{1}}+R_{2}\right)$,

where $i_{p o l, i}$ and $i_{p o l, f}$ are the initial and final currents flowing into the VSC during the following time step, respectively. The same with $k+1$ current, $v_{C}$ at $k+1$ instant should be estimated under all potential $v_{i}$ for $\mathrm{CF}$ of which the following subsection will give details.

\section{B. Stabilization via Cost Function}

As shown in Fig. 7, the FCS-MPC algorithm is ended with the minimization of $\mathrm{CF}$ which determines the vi to be applied at the next instant. The $\mathrm{CF}$ consists of the errors between the predicted voltage/current values and their references; further, the current limiting term $G_{l i m}$ is also included:

$$
G_{\text {lim }}=\left\{\begin{array}{l}
0, \text { if }\left|\bar{i}_{o}\right| \leq i_{\max } \\
\infty, \text { if }\left|\bar{i}_{o}\right|>i_{\max }
\end{array} .\right.
$$

The control term for ac current regulation is given in a conventional way, as:

$$
g_{\text {con }}=\left(i_{o \alpha}^{*}-i_{o \alpha}\right)^{2}+\left(i_{o \beta}^{*}-i_{o \beta}\right)^{2} .
$$

Lastly, both two control terms are integrated into a total cost function:

$$
g_{t}=g_{c o n}+\lambda_{d c} g_{C}+G_{l i m}
$$

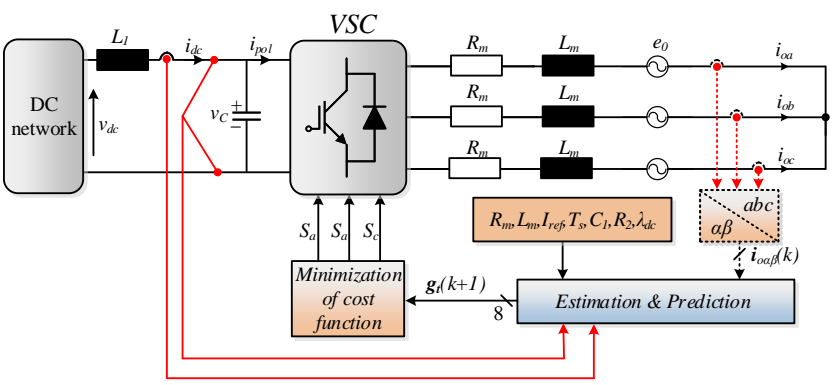

Fig. 7. Complete implementation of stabilization approach

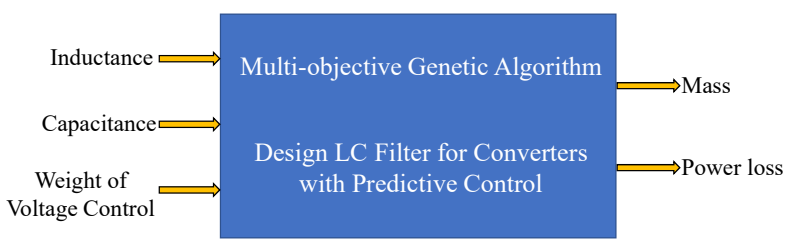

Fig. 8. MOOP of $L C$ filter in aircraft actuation system.

where

$$
g_{C}=\left(v_{C}^{*}-v_{C}\right)^{2} .
$$

Here, $\lambda_{d c}$ is the weighting factor of dc voltage control term. As this work focuses on the control preference between $v_{C}$ and $i_{o}$, other control terms (e.g. derivative of the voltage reference, switching penalization) are not considered here thus there is only one WF in $g_{t}$. In contrast to [18], [38], $\lambda_{d c}$ in this study is set as a design variable in the optimization. It is supervised by GA objectives but more importantly the feasible design point must provide good control performance. Only the potential design, that meets various performance constraints in GA optimization, can be used to give a feasible filter design.

An attractive feature of FCS-MPC is that no modulation stage is needed, but it will cause a variant switching frequency which may affect the steady-state performance [42]. This paper only utilizes the conventional FCS-MPC with the dc stabilization term in (14) for the sake of simplicity, but it is stable enough according to the Nyquist stability criterion [38]. Improved FCS-MPC approaches with PWM can be found in [41], [42]. The following two sections will introduce the application of $2 \mathrm{SA}$ on the $L C$ filter optimization.

\section{GA Optimization - SeArch Algorithm}

Fig. 8 briefly depicts the MOOP of filter in the aircraft actuator with three DVs $\left(L_{1}, C_{1}\right.$ and $\lambda_{d c}$; their ranges are in Table III) and two objectives (filter Mass $M$ and Power Loss $P L)$. In addition, multi-objective GA is utilized as the search algorithm to find the best solution of this MOOP based on the filter sizing models. The following two subsections will first give the GA optimization process and then discuss the optimization results.

\section{A. GA Optimization Process}

As shown in Fig. 9, to start the $L C$ filter design optimization process, the first generation of chromosomes needs to be 
TABLE III

DV RANGES IN OPTIMIZATION

\begin{tabular}{cc}
\hline \hline DVs & Ranges \\
\hline Inductance $L_{1}$ & {$[1,100] \mu H$} \\
Capacitance $C_{1}$ & {$[1,2000] \mu F$} \\
Weighting Factor $\lambda_{d c}$ & {$[0.1,10]$} \\
\hline \hline
\end{tabular}

randomly created after setting the GA operator parameters including selection, crossover and mutation fractions. In the commercial GA Matlab tool, users can set different values to these parameters but here default values of these parameters are used for the sake of convenience. Besides, Pareto fraction [17], [22] should also be confirmed for the multi-objective optimization. Noting that the GA tool utilized here is a controlled, elitist genetic algorithm (a variant of NSGA-II [28]). NSGA III [29] is a more recent variation of the GA; however all of them are focusing on multi-objective optimization problems and using bio-inspired operators to produce the next population thus should be the search algorithms in 2SA. NSGA-II applies a fast non-dominating ranking method and an extra niching operator to deal with multi-objective problems [28]; NSGA-III uses the same framework with NSGA-II but works with a set of supplied or predefined reference points [29], [30].

Fitness of individuals (parents and children) here consist of the values of two objectives whose weights are set equally for GA operation. Fig. 9 summarizes the overall optimization procedure. The upper rectangle part of Fig. 9 shows the running function of "gamultiobj" in Matlab [43]; the below rectangle depicts the main function of this study which is case-specfic with the target MOOP. In the running function, a new generation of chromosomes (children) which maps the variables can be created by the three operators after ranking the fitness of the current generation (parents). If the iteration times does not exceed the pre-set maximum generation number, individuals (only know values of three variables) of children should go to the main function to get their objective values until reaching the maximum generation number. In the main function, objectives and their fitness of the new generation (children) should be obtained by the integrated filter sizing model (Fig. 5) for the next selection of chromosome population (children of children). However, every individual should be checked by all constraints including sizing constraints (Fig. 5) and circuit constraints (Table IV). If constraints violate a design, their objectives will be assigned the infinite values and this design will stay at the bottom of the fitness ranking in the new generation. If not, filter mass and power loss of this design can be given as its two objectives.

\section{B. Constraints and Parameters in GA Optimization}

As shown in Fig. 9, for each filter design candidate the resulting signals must be compared with the various constraints before being a feasible design. All the circuit constraints are summarized in Table IV. Regarding the $v_{d c}$ transient and distortion spectrum constraints, upper or lower limits are provided by [44] and the simulation signals can be analyti-

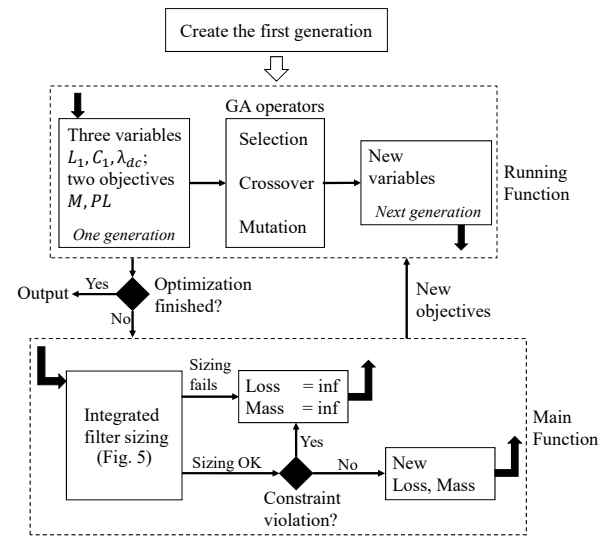

Fig. 9. Optimization procedure based on multi-objective GA.

cally processed and compared with them in the optimization process. The DC distortion factor is the ratio of the DC distortion to the DC steady state voltage and its maximum value is set as 0.015 for 270 Volt DC system. The other constraint in [44] for $v_{d c}$ is the maximum voltage ripple, 6 Volt. Besides, the maximum tracking error and THD settings are given by trial-and-error which should be specific for the target MOOP. In order to embed THD calculation into the automated GA optimization, this study utilizes Matlab codes to implement the Discrete Fourier Transformation (DFT) [45]. The circuit parameters are also given in Table IV, including the DC voltage, impedance and AC load parameters. The sampling time is set as $25 \mu \mathrm{s}$, peak values of $e_{0}$ and $i_{o}$ are $100 \mathrm{~V}$ and $10 \mathrm{~A}$, the AC frequency is set as $50 \mathrm{~Hz}$ [39].

The sizing and GA parameters (unchanged during the optimization) are summarized in Table IV. The $L C$ filter sizing is based on the commercial data from manufactures: $A_{L}, l_{e}, A_{c, \text { min }}, A_{c, s}$ etc., which are specific to different inductor cores, are all recorded from [36]; besides, capacitor mass, volume, $I_{C, \max }$ and $R_{t h}$ are from [37]. The options in GA optimization are using default settings in [43] except for Pareto fraction, population size and the max generation number in Table IV. Furthermore, the linear equality/inequality constraints as well as nonlinear constraint function are not edited in the running function of "gamultiobj" because, as shown in Fig. 9, all constraint violations are processed by setting infinite values to GA objectives, i.e. hard constraints for both sizing constraints (Fig. 5) and circuit constraints given in Table IV.

\section{GA Optimization Results}

This section reports the optimization results obtained from running the multi-objective GA with a population size 300 and number of generations 100, where 21734 designs are violated by constraints and 8566 designs are feasible. Pareto-optimal solutions of filter designs are generated after which the design distribution of $L_{1}$ and $C_{1}$ during the optimization process is shown with an integrated index of two objectives. Then, the design distribution of failure sizing points is extracted to show the distribution regularities and the violation situations of different constraints. Finally, the study on weighting factor 
TABLE IV

Constraints And Parameters in FILTER Design ANd Optimization

\begin{tabular}{lclc}
\hline \hline Circuit Constraints & \multicolumn{3}{l}{ Circuit Parameters } \\
\hline$v_{d c}$ voltage transient & Fig. 16 in [44] & Supply voltage & $270 \mathrm{~V}$ \\
$v_{d c}$ distortion spectrum & Fig. 18 in [44] & MG impedance & $L_{0}=1 \mu \mathrm{H}, R_{0}=1 \mathrm{~m} \Omega$ \\
Max. distortion factor of $v_{d c}$ & 0.015 & AC load & $L_{m}=2.5 \mathrm{mH}, R_{m}=10 \Omega$ \\
Max. voltage ripple of $v_{d c}$ & $6 \mathrm{~V}$ & Peak back-EMF & $100 \mathrm{~V}$ \\
Max. tracking error of $i_{o}$ & $0.35 \mathrm{~A}$ & Sampling time $T_{s}$ & $25 \mu \mathrm{s}$ \\
Max. THD of $i_{o}$ & $6 \%$ & Current reference & $I_{\text {ref }}=10 \mathrm{~A}, f_{\text {ref }}=50 \mathrm{~Hz}$ \\
\hline Sizing Parameters & $5 \times 10^{-5} \mathrm{~m}$ & GA Parameters & \\
\hline$h_{\text {ins }}$ & $8 \times 10^{6} \mathrm{~A} / \mathrm{m}^{2}$ & Crossover fraction & 0.8 \\
$J_{\max }$ & 0.8 & Mute count & $0.05 \times 300$ \\
$\gamma_{\max }$ & $240 \pi \times 10^{-7} \mathrm{H} / \mathrm{m}$ & Pareto fraction & 0.01 \\
$\mu$ & $3.75 \times 10^{4} \mathrm{At} / \mathrm{m}$ & Population size & 0.5 \\
$H_{\text {max }}$ & $5.7 \times 10^{7} \mathrm{~S}$ & Max. generation No. & 300 \\
$L_{1}$ wire conductivity & $1.02 \mathrm{~g} / \mu \mathrm{F}$ & & 100 \\
$C_{1}$ linear ratio for $M$ & \multicolumn{4}{c}{} \\
\hline \hline
\end{tabular}

TABLE V

Best Design Point in GA Optimization

\begin{tabular}{lc}
\hline \hline Inductor inductance $L_{1}$ & $21.1 \mu \mathrm{H}$ \\
Inductor resistance $R_{1}$ & $13.86 \mathrm{~m} \Omega$ \\
Capacitor capacitance $C_{1}$ & $306 \mu \mathrm{F}$ \\
Capacitor resistance $R_{2}$ & $12.48 \mathrm{~m} \Omega$ \\
Weighting factor $\lambda_{d c}$ & 3.3586 \\
Power loss $P L$ & $0.9431 \mathrm{~W}(\max .4 .338 \mathrm{~W})$ \\
Mass $M$ & $0.5275 \mathrm{~kg}(\max .2 .289 \mathrm{~kg})$ \\
$r$ value & 0.3168 \\
\hline
\end{tabular}

$\left(\lambda_{d c}\right)$ is exercised to demonstrate the trade-off function of this factor in system stabilization.

1) Optimal Results

The optimal design results using GA are represented in Fig. 10. Fig. 10(a) shows the distributions of feasible design points which are marked by multi-colored circles. The color corresponds to the value of the integrated index $(r)$ of two objectives which is utilized to select one particular point for each Pareto front. In contrast to the analytic hierarchy process (AHP) employed in [3], the criterion for this decision-making solution is the minimal distance from ideal objectives [4]:

$$
\text { Solution } \equiv \min \left(r_{i}\right)
$$

where

$$
r_{i}=\sqrt{\left(\frac{M_{i}}{M_{\max }}\right)^{2}+\left(\frac{P L_{i}}{P L_{\max }}\right)^{2}},
$$

$M_{i}$ : Mass of the $i$ th solution;

$M_{\max }$ : Maximum mass;

$P L_{i}$ : Power loss of the $i$ th solution;

$P L_{\max }$ : Maximum power loss.

As can be seen from the color distribution, there is a decreased trend of $r$ value as the inductance and capacitance reduce. The design points in Pareto front are also shown in the figure. However, they are not all good solutions according to the $r$-value criterion. The best design point chosen by (16), (17) is presented in Table $\mathrm{V}$ together with the inner resistance and the final $r$ value. Fig. 10(b) depicts the two objectives of feasible design points where the Pareto-optimal design configuration is generated. For the motor optimization problems in [4], equality constraints are added for the solution distribution of Pareto front to ensure that there is no vacancy between the solutions in two different system levels. Conversely, in this paper, the final Pareto solution is not processed by equality constraints; therefore, there are data gaps among the Pareto-front points and we can see that these solutions use different cores (No. 16 and No. 17 in [36]) for inductor sizing which also contributes to their big weight differences. Another reason of data gap in the Pareto front is from the various GA constraints (see Section V.B) which limit a lot of potential design points from the Pareto front.

2) Design Points Filtered Out by Constraints

The failure design points within the optimization process are displayed in Fig. 11(a) with their failure reasons. No design point is filtered out by the dc voltage constraint. Most of design points are violated by the THD limitation of ac current. It is interesting to note that there is a lower capacitance bound for these points. Besides, many points are deleted from the selection pool since they violate both constraints of ac current while only few points are eliminated due to excessive current errors. Furthermore, the max-number of inductor iteration $(N)$ is set as 10 and the inductance of failure points filtered out by this reason mostly stays around $21.1 \mathrm{mH}$. Lastly, there are only two design points failing with the limit of capacitor temperature rise.

It is noted that the constraint check order can affect the distribution of these failure design points because the violation reminders are returned by the first constraint check. Namely, once a constraint is violated, the following constraints will not be checked. The check order used in this study is: ac current constraint, integrated sizing function and de voltage constraints. 


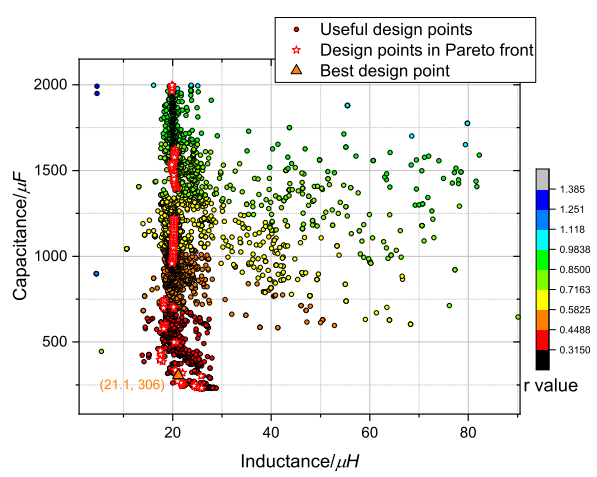

(a) Design distribution of $L_{1}, C_{1}$.

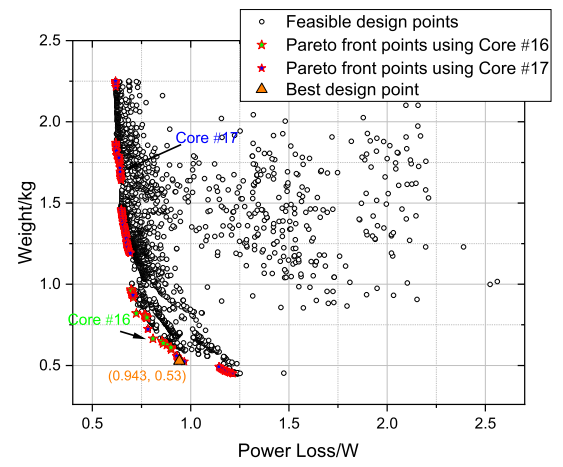

(b) Feasible design points and the Pareto front.

Fig. 10. Optimal design results based on multi-objective GA.

3) Study on Weighting Factor

As discussed in Section V, two current constraints are extremely sensitive to the optimization results and this phenomenon can be easily seen from Fig. 11(a) where the vast majority (more than $99 \%$ ) of failure design points are filtered out by these two limits. This section investigates the sensibility of both limits with regards to $\lambda_{d c}$ based on the best design point, as shown in Fig. 11(b).

Using the inductance and capacitance of the best design point, give $\lambda_{d c}$ sample values in the interval $[0.1,10]$ followed by running simulation to record the average current error with the reference (blue line) and the THD value (red line) of ac current. The current difference is always under the upper limit $(0.35 A)$ if $\lambda_{d c}$ is smaller than 4. However, THD is more sensitive because only two of these 30 sample points can meet the THD constraint: no larger than $6 \%$. One interesting thing comes from the comparison of the best design point and the two sample points around it. Regarding both current difference and THD, the best point is smaller than any of these sample points; more importantly, the two sample points are not able to satisfy the THD limitation while the THD value of the best design point (5.994\%) was just a little smaller than the limit, which demonstrates a very good searching ability of GA operators.

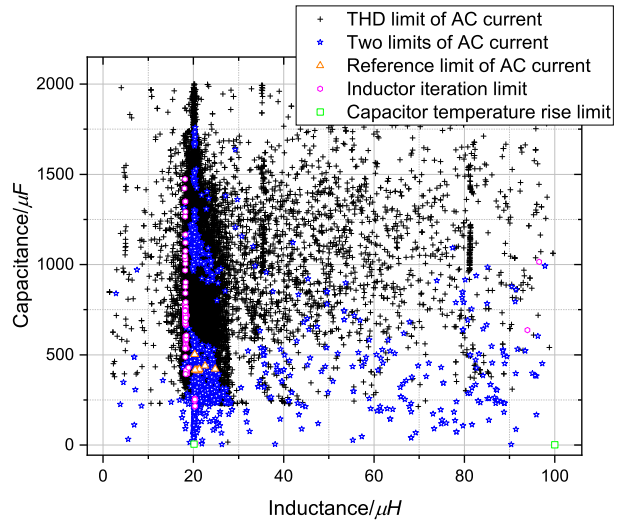

(a) Failure design points.

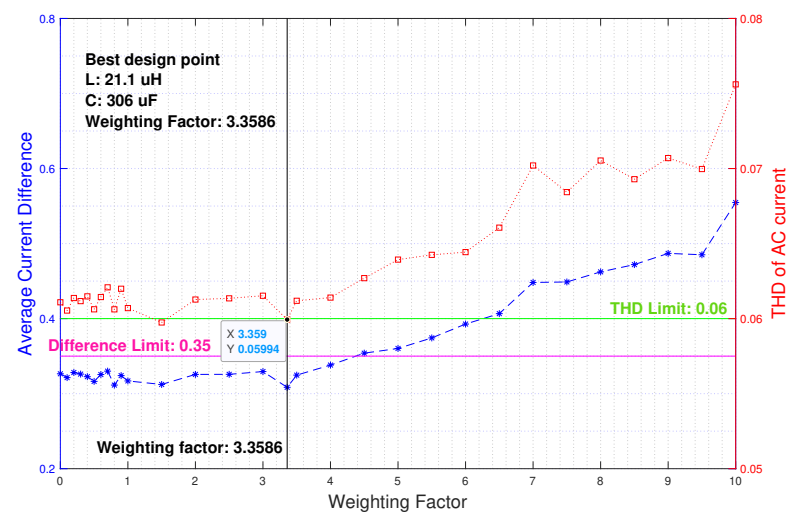

(b) Sensibility analysis of weighting factor $\lambda_{d c}$.

Fig. 11. Failure designs in optimization and sensibility analysis of $\lambda_{d c}$.

\section{ML TRAining AND Optimization - SuRRogate ALGORITHM}

After the GA optimization, this study utilizes two ML methods to explore an alternative for the same MOOP. Firstly, a generic ML structure is proposed which should be available for all optimization problems (no matter what optimization algorithm it is using); Then, SVM is employed to address constraints the surrogate model; Finally, ANN is applied for the mapping surrogate from design variables to objective(s).

\section{A. ML Optimization Structure}

As shown in Fig. 12, a generic ML optimization structure is proposed with two different ML models for the studied filter MOOP. The first model is for constraint violation, namely it is used for finding which data in design variable space should be feasible design according to the aforementioned various constraints; After that, feasible designs are regarded as the input of the second ML model to bridge the gap between design variables and objectives. ANN is suggested for this mapping function.

Both of two models are obtained from superivised learning which means input-output data are required for their training. In this paper, 30300 sample data set (all sampling designs in GA optimization) is given for training the constraint violation 


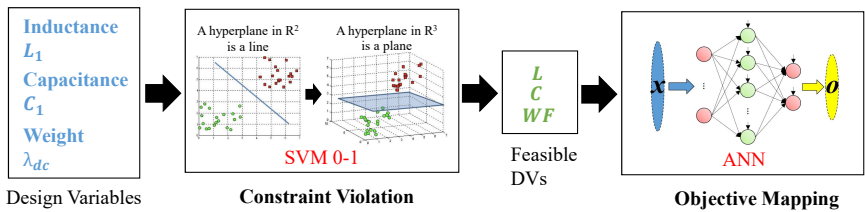

(DVs)

Fig. 12. Structure of the ML based optimization.

model while only 8566 feasible designs are used to train the ANN model.

\section{B. Support Vector Machine for Constraint Violation}

SVM is a discriminative classifier formally defined by training a separating hyperplane [20] with the maximum distance from the nearest training data. Given labelled training data (the output is 1 for one feature and 0 for the other feature), the algorithm outputs an optimal hyperplane which can categorize new samples. In a 3D space, this hyperplane is a plane separating the space in two parts for feature classification (see the SVM block in Fig. 12).

In this study, raw data for SVM is labelled with 0-1; feasible designs have 1 as the output and failure designs have 0 . After that, the input should be processed by normalization in order to get better training performance. Then, the supervised learning is implemented by 'fitcsvm' function [46] in Matlab where 'KernelFunction' is set as 'rbf' (radial basis function). In addition, 'KernelScale' is set as 'auto', 'Standardize' is 'ture' and 'outlierFraction' is zero [20], [46]. It takes 102.95 secs to train this SVM model in Matlab on a standard computer and around 8 mins to do the further cross-validation [33] and validation loss calculation. The training performance is quantified by the class loss which can be obtained using crossvalidation [33]. In this study, the class loss result is $7.53 \%$. The authors also tried different ML algorithms for this constraint violation model but no better results obtained. For example, ANN Fitting was first utilized for this classification training with the outputs processed to 0-1; However, the accuracy of ANNs with various structures is always below 89\%. We also trained this classification problem using ANN Patter Recognition (PR) but the accuracy results cannot exceed $88 \%$. Therefore, the trained SVM model is utilized as the final constraint violation model for the studied ML optimization problem.

\section{Artificial Neural Network for Performance Mapping}

1) Mapping Two Objectives

After training the SVM surrogate model, two different ANNs are constructed by using 4-layer networks to do the mapping, marked as $A N N_{1}$ and $A N N_{2} . A N N_{1}$ maps two objectives (mass and power loss) to the three design variables. In particular, this network represents the following relation:

$$
y=F(x) \Leftrightarrow(M, P L)=F_{1}\left(L_{1}, C_{1}, \lambda_{d c}\right) .
$$

An integrated mapping surrogate network is built instead of deploying two independent ANNs for two objectives.

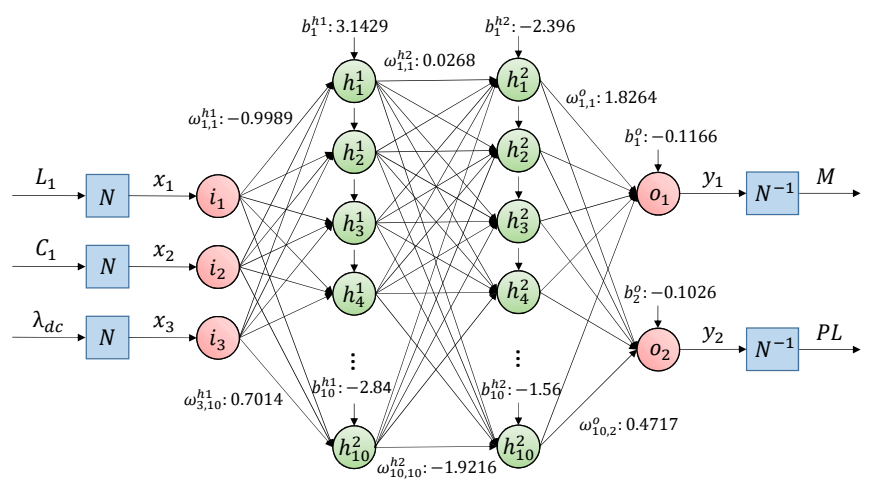

(a) Mapping two objectives.

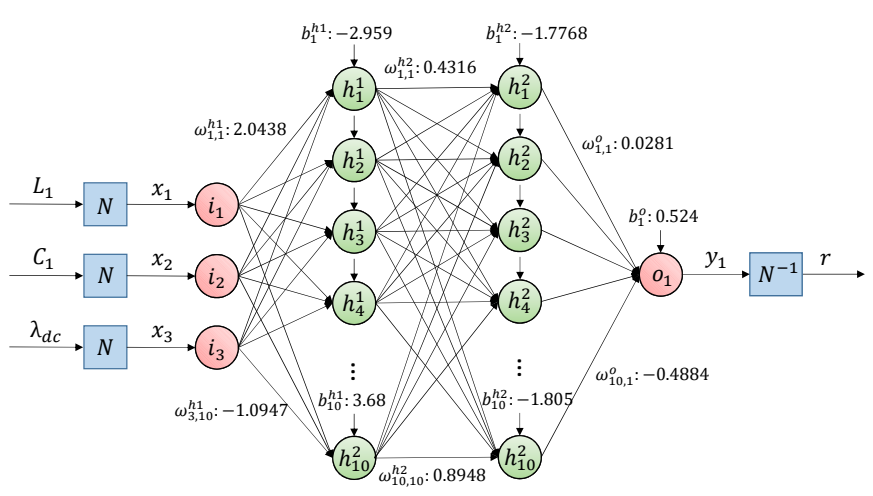

(b) Mapping one objective.

Fig. 13. Deployment of the ANN surrogate models with normalization functions at the inputs and de-normalization functions at the output.

As shown in Fig. 13(a), $A N N_{1}$ has two hidden layers whose neurons are marked in green. The first and last weights between each layer as well as biases in three layers are displayed, other weights and biases are omitted for simplicity.

In order to compare the prediction performance of two different networks, except setting the same neuron numbers (10 neurons) in the hidden layers, both $A N N_{1}$ and $A N N_{2}$ are using Bayesian Regularization training function [47]. And the maximum epochs for training is both set 500 , learning rate is set as 0.1 . The $A N N_{1}$ training process costs 18.08 secs with 500 epochs. The predict performance for Mass (RMSE) is $0.003 \mathrm{~kg}$; And for power loss prediction, the error is $0.0197 \mathrm{Watt}$.

2) Mapping One Objective

In contrast, $A N N_{2}$ maps just one objective ( $r$ value, Eq. (17)) to the three design variables. In particular, this network represents the following relation:

$$
y=F(x) \Leftrightarrow(r)=F_{2}\left(L_{1}, C_{1}, \lambda_{d c}\right)
$$

Fig. 13(b) shows the deployment of $A N N_{2}$. The same with $A N N_{1}$, only fringe weights and biases are depicted, other weights and biases are omitted for simplicity. The $A N N_{2}$ training process costs 11.57 secs with 500 epochs. The prediction performance for $r$ value (RMSE) is only 0.0019 .

3) Optimization Using Machine Learning Models 


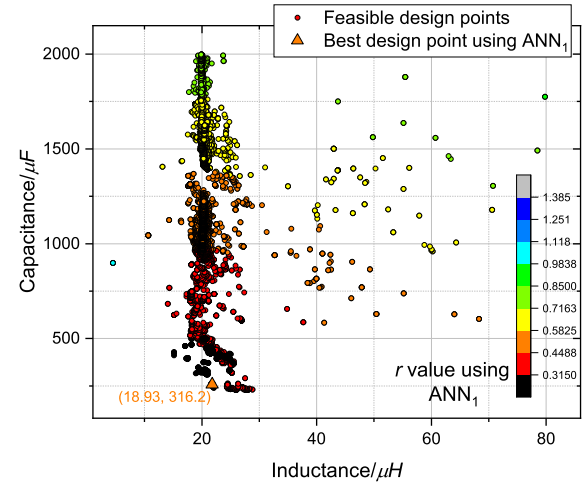

(a) Mapping two objectives, $A N N_{1}$.

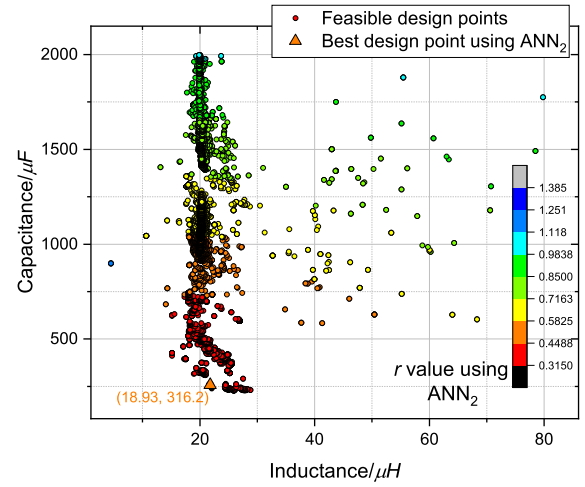

(b) Mapping one objective, $A N N_{2}$.

Fig. 14. Optimal design results using two ANNs.

Following two different ANN designs above, this subsection will give the optimization results based on the trained ML models and comparisons with GA results are also discussed. Fig. 14 shows the distributions of $r$ value using above ML models. Noting that SVM model is the same in the proposed generic surrogate structure while neural networks are $A N N_{1}$ and $A N N_{2}$ respectively. Therefore, the corresponding distributions of $r$ value varies. Two figures in Fig. 14 are using the same color map scale with Fig. 10(a) (raw data). Thus the difference of $r$ value distributions is obvious. Fig. 14(b) approximates the GA optimization results much closer than Fig. 14(a).

The best-design results obtained from the proposed " $2 \mathrm{SA}$ " are summarized in Table VI. It is interesting to notice that the inputs of best design points obtained from two ANNs are the same and both close to the GA optimization. Both $A N N_{1}$ and $A N N_{2}$ give good prediction performance with regards to their specfic output(s) based on the trained SVM model. But $A N N_{1}$ performs much worse than $A N N_{2}$ on $r$ value prediction: RMSE, which is computed by comparing all raw data and their predictions, is much larger in $A N N_{1} . A N N_{1}$ can predict close values for both Mass and Power Loss: RMSE for $M$ is 0.003 $k g$ and RMSE for $P L$ is $0.0197 \mathrm{~W}$. However, after using Eq. (17), $r$ value becomes quite different from raw data because $A N N_{1}$ gives relatively larger $P L$ but smaller $M$. Therefore, the second way of building ANN should be better for potential MOOPs which has an index $(r$
TABLE VI

Best Design Point Using ML Models

\begin{tabular}{lccc}
\hline \hline Terms of design & $A N N_{1}$ & $A N N_{2}$ & GA results \\
\hline$L_{1}(\mu H)$ & 18.93 & 18.93 & 21.1 \\
$C_{1}(\mu F)$ & 316.23 & 316.23 & 306 \\
$\lambda_{d c}$ & 3.4162 & 3.4162 & 3.3586 \\
$P L(W)$ & 0.9337 & - & 0.9431 \\
$M(k g)$ & 0.5349 & - & 0.5275 \\
$r$ & 0.282 & 0.3177 & 0.3168 \\
RMSE $-r$ value & $\mathbf{0 . 1 1 9 7 6}$ & $\mathbf{0 . 0 0 1 8 8 4}$ & - \\
\hline \hline
\end{tabular}

value) integrating one or more optimization objectives. It is noted that above RMSE values are given by all raw data. In training process of both $A N N_{1}$ and $A N N_{2}$, there are 8566 raw data points. This data set was then randomly divided into three data sets, i.e. the training set $(70 \%$ of data, corresponding to 5996 data points), the validation set (15\% of data, corresponding to 1285 data points) and the testing set (15\% of data, corresponding to 1285 data points). Training data is used to directly modify weight and bias values; validation data is used for validating stopping condition and ranking of net candidates; testing data is used to obtain unbiased estimates of non-training data. Therefore, only $70 \%$ of raw data are directly used for training ANN, other $30 \%$ of raw data are new for the trained networks. For $A N N_{2}$, the RMSE of training data is 0.00143 and the RMSE of non-training (validation \& test) data is 0.00205 .

As discussed above, the training time of SVM is 102.95 secs. Further, on a standard desktop computer, the training of $A N N_{2}$ takes 11.57 secs. In the prediction process, the running time of trained SVM and $A N N_{2}$ takes 23.88 and 0.55 secs, respectively. Therefore, the total time of using surrogate algorithms is 138.95 secs. In contrast, running detailed simulations in GA optimization is several orders of magnitude slower. It costs around 3.2 secs to obtain one design point, therefore taking more than 20 hours to do the GA optimization with population size of 300 and number of generations of 100. The efficiency capability makes surrogate algorithms a very promising approach for the potential filter MOOP tasks.

\section{CONCLUSION}

This paper proposes a novel category approach (2SA model) for the commonly used algorithms in multi-discipline field and initially explores the application of 2SA for the fitler optimization in aircraft actuation system. The optimization process and results of search algorithm (GA) are first discussed followed by using two surrogate algorithms (SVM \& ANN) to train the GA data and predict the filter performance. Comparison shows that surrogate models can give good prediction performance but the objective mapping model should be carefully selected since different objectives are usually competing which may generate bad predictions. 


\section{ACKNOWLEDGMENT}

This project has received funding from the Clean Sky 2 Joint Undertaking under the European Union's Horizon 2020 research and innovation programme under grant agreement No 807081.

\section{REFERENCES}

[1] P. Wheeler and S. Bozhko, "The more electric aircraft: Technology and challenges." IEEE Electrific. Mag., vol. 2, no. 4, pp. 6-12, 2014.

[2] F. Gao, S. Bozhko, A. Costabeber, G. Asher, and P. Wheeler, "Control design and voltage stability analysis of a droop-controlled electrical power system for more electric aircraft," IEEE Trans. Ind. Electron., vol. 64, no. 12, pp. 9271-9281, 2017.

[3] Z. Jiao, B. Yu, S. Wu, Y. Shang, H. Huang, Z. Tang, R. Wei, and C. Li, "An intelligent design method for actuation system architecture optimization for more electrical aircraft," Aerospace Science and Technology, vol. 93, p. 105079, 2019.

[4] H. Ounis, B. Sareni, X. Roboam, and A. De Andrade, "Multi-level integrated optimal design for power systems of more electric aircraft," Mathematics and Computers in Simulation, vol. 130, pp. 223-235, 2016.

[5] J. A. Rosero, J. A. Ortega, E. Aldabas, and L. Romeral, "Moving towards a more electric aircraft," IEEE Aerosp. Electron. Syst. Mag., vol. 22, no. 3, pp. 3-9, 2007.

[6] D. Salomonsson and A. Sannino, "Low-voltage de distribution system for commercial power systems with sensitive electronic loads," IEEE Trans. Power Del, vol. 22, no. 3, pp. 1620-1627, 2007.

[7] B. Sarlioglu and C. T. Morris, "More electric aircraft: Review, challenges, and opportunities for commercial transport aircraft," IEEE Trans. Transport. Electrific., vol. 1, no. 1, pp. 54-64, 2015.

[8] F. Gao and S. Bozhko, "Modeling and impedance analysis of a single dc bus-based multiple-source multiple-load electrical power system," IEEE Transactions on Transportation Electrification, vol. 2, no. 3, pp. 335346, 2016.

[9] F. Gao, X. Zheng, S. Bozhko, C. I. Hill, and G. Asher, "Modal analysis of a pmsg-based dc electrical power system in the more electric aircraft using eigenvalues sensitivity," IEEE Trans. Transport. Electrific., vol. 1, no. 1 , pp. $65-76,2015$.

[10] F. Wang, G. Chen, D. Boroyevich, S. Ragon, M. Arpilliere, and V. R. Stefanovic, "Analysis and design optimization of diode front-end rectifier passive components for voltage source inverters," IEEE Trans. Power Electron., vol. 23, no. 5, pp. 2278-2289, 2008.

[11] B. Cheong, "Advanced modelling and evolutionary multiobjective optimization of motor drive for aerospace applications," Ph.D. dissertation, Department of Electrical and Electronic Engineering, University of Nottingham, 2019.

[12] A. Kouchaki and M. Nymand, "Analytical design of passive lcl filter for three-phase two-level power factor correction rectifiers," IEEE Trans. Power Electron., vol. 33, no. 4, pp. 3012-3022, 2018.

[13] J. Muhlethaler, M. Schweizer, R. Blattmann, J. W. Kolar, and A. Ecklebe, "Optimal design of lcl harmonic filters for three-phase pfc rectifiers," IEEE Trans. Power Electron., vol. 28, no. 7, pp. 3114-3125, 2013.

[14] J. Muhlethaler, J. W. Kolar, and A. Ecklebe, "Loss modeling of inductive components employed in power electronic systems," in 8th International Conference on Power Electronics - ECCE Asia, pp. 945-952, 2011.

[15] Y. Jiao and F. C. Lee, "Lcl filter design and inductor current ripple analysis for a three-level npc grid interface converter," IEEE Transactions on Power Electronics, vol. 30, no. 9, pp. 4659-4668, 2014.

[16] E. K. Burke, G. Kendall et al., Search methodologies. Springer, 2005.

[17] B. Sareni, J. Regnier, and X. Roboam, "Recombination and selfadaptation in multi-objective genetic algorithms," in International Conference on Artificial Evolution (Evolution Artificielle), pp. 115-126. Springer, 2003.

[18] T. Dragicevic and M. Novak, "Weighting factor design in model predictive control of power electronic converters: An artificial neural network approach," IEEE Trans. Ind. Electron., pp. 1-1, 2018.

[19] T. Dragičević, P. Wheeler, and F. Blaabjerg, "Artificial intelligence aided automated design for reliability of power electronic systems," IEEE Transactions on Power Electronics, vol. 34, no. 8, pp. 7161-7171, 2018.

[20] Z. Li, R. Outbib, S. Giurgea, D. Hissel, S. Jemei, A. Giraud, and S. Rosini, "Online implementation of svm based fault diagnosis strategy for pemfc systems," Applied energy, vol. 164, pp. 284-293, 2016.
[21] J. Liu, Q. Li, W. Chen, Y. Yan, and X. Wang, "A fast fault diagnosis method of the pemfc system based on extreme learning machine and dempster-shafer evidence theory," IEEE Transactions on Transportation Electrification, vol. 5, no. 1, pp. 271-284, 2018.

[22] K. Wang and B. Zhang, "Multiobjective trajectory optimization for a suborbital spaceplane using directed search domain approach," Aerospace Science and Technology, vol. 77, pp. 713-724, 2018.

[23] J. Shen and A. Khaligh, "A supervisory energy management control strategy in a battery/ultracapacitor hybrid energy storage system," IEEE Transactions on Transportation Electrification, vol. 1, no. 3, pp. $223-$ $231,2015$.

[24] L. Zhang, Z. Zhou, Q. Chen, R. Long, and S. Quan, "Model predictive control for electrochemical impedance spectroscopy measurement of fuel cells based on neural network optimization," IEEE Transactions on Transportation Electrification, vol. 5, no. 2, pp. 524-534, 2019.

[25] A. G. Sarigiannidis, M. E. Beniakar, P. E. Kakosimos, A. G. Kladas, L. Papini, and C. Gerada, "Fault tolerant design of fractional slot winding permanent magnet aerospace actuator," IEEE Transactions on Transportation Electrification, vol. 2, no. 3, pp. 380-390, 2016.

[26] A. Sarkheyli, A. Bagheri, B. Ghorbani-Vaghei, and R. AskariMoghadam, "Using an effective tabu search in interactive resources scheduling problem for leo satellites missions," Aerospace Science and Technology, vol. 29, no. 1, pp. 287-295, 2013.

[27] M. Jafari, L. E. Brown, and L. Gauchia, "Hierarchical bayesian model for probabilistic analysis of electric vehicle battery degradation," IEEE Transactions on Transportation Electrification, vol. 5, no. 4, pp. 12541267, 2019.

[28] K. Deb, A. Pratap, S. Agarwal, and T. Meyarivan, "A fast and elitist multiobjective genetic algorithm: Nsga-ii," IEEE transactions on evolutionary computation, vol. 6, no. 2, pp. 182-197, 2002.

[29] H. Seada and K. Deb, "U-nsga-iii: A unified evolutionary algorithm for single, multiple, and many-objective optimization," COIN report, vol. 2014022, 2014

[30] K. Deb and H. Jain, "An evolutionary many-objective optimization algorithm using reference-point-based nondominated sorting approach, part i: solving problems with box constraints," IEEE transactions on evolutionary computation, vol. 18, no. 4, pp. 577-601, 2013.

[31] R. Norouzi, A. Kosari, and M. H. Sabour, "Real time estimation of impaired aircraft flight envelope using feedforward neural networks," Aerospace Science and Technology, vol. 90, pp. 434-451, 2019.

[32] R. Thirumalainambi and J. Bardina, "Training data requirement for a neural network to predict aerodynamic coefficients," in Independent Component Analyses, Wavelets, and Neural Networks, vol. 5102, pp. 92-103. International Society for Optics and Photonics, 2003.

[33] Z. Xu, Y. Gao, X. Wang, X. Tao, and Q. Xu, "Surrogate thermal model for power electronic modules using artificial neural network," in IECON 2019-45th Annual Conference of the IEEE Industrial Electronics Society, vol. 1, pp. 3160-3165. IEEE, 2019.

[34] T. P. Lillicrap, J. J. Hunt, A. Pritzel, N. Heess, T. Erez, Y. Tassa, D. Silver, and D. Wierstra, "Continuous control with deep reinforcement learning," arXiv preprint arXiv:1509.02971, 2015.

[35] A. Boutemedjet, M. Samardžić, L. Rebhi, Z. Rajić, and T. Mouada, "Uav aerodynamic design involving genetic algorithm and artificial neural network for wing preliminary computation," Aerospace Science and Technology, vol. 84, pp. 464-483, 2019.

[36] Magnetics, "2017 magnetics powder core catalog - toroid data," May. 2017. [Online]. Available: https://www.mag-inc.com/Company/News/2017\%E5\%B9\% B411\%E6\%9C\%88/2017-Magnetics-Powder-Core-Catalog-in-Chinese

[37] Capacitor Film, "Power electronic capacitors, film capacitor mkp dc b2562*," May. 2018. [Online]. Available: https://en.tdk-electronics.tdk. com/inf/20/50/ds/B2562_.pdf

[38] T. Dragicevic, "Dynamic stabilization of dc microgrids with predictive control of point-of-load converters," IEEE Trans. Power Electron., vol. 33, no. 12, pp. 10872-10 884, 2018.

[39] J. Rodriguez, J. Pontt, C. A. Silva, P. Correa, P. Lezana, P. Cortés, and U. Ammann, "Predictive current control of a voltage source inverter," IEEE transactions on industrial electronics, vol. 54, no. 1, pp. 495-503, 2007.

[40] F. De Bosio, L. A. de Souza Ribeiro, F. D. Freijedo, M. Pastorelli, and J. M. Guerrero, "Effect of state feedback coupling and system delays on the transient performance of stand-alone vsi with lc output filter," IEEE Transactions on Industrial Electronics, vol. 63, no. 8, pp. 4909-4918, 2016.

[41] Z. Ma, X. Zhang, J. Huang, and B. Zhao, "Stability-constrainingdichotomy-solution-based model predictive control to improve the sta- 
bility of power conversion system in the mea," IEEE Transactions on Industrial Electronics, vol. 66, no. 7, pp. 5696-5706, 2018.

[42] C. Zheng, T. Dragičević, B. Majmunović, and F. Blaabjerg, "Constrained modulated model-predictive control of an lc-filtered voltage-source converter," IEEE Transactions on Power Electronics, vol. 35, no. 2, pp. 1967-1977, 2019.

[43] "gamultiobj - find pareto front of multiple fitness functions using genetic algorithm," 2020. [Online]. Available: https://uk.mathworks. com/help/gads/gamultiobj.html

[44] Military Standard, "Aircraft electric power characteristics," Department of Defense Interface Standard (MIL-STD-704F), 2004.

[45] Y. Levron, "Computes the total harmonic distortion (thd) of a signal, " Jul. 2020. [Online]. Available: https://uk.mathworks.com/matlabcentral/fileexchange/ 40455-computes-the-total-harmonic-distortion-thd-of-a-signal

[46] "fitcsvm - train support vector machine (svm) classifier for oneclass and binary classification," 2020. [Online]. Available: https: //uk.mathworks.com/help/stats/fitcsvm.html

[47] "trainbr -bayesian regularization backpropagation," 2020. [Online]. Available: https://uk.mathworks.com/help/deeplearning/ref/trainbr.html

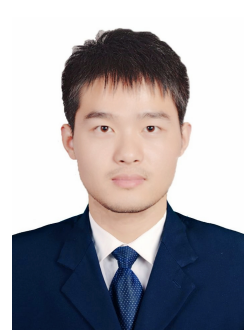

Yuan Gao (S'19) is currently pursuing the Ph.D. degree of Electrical and Electronic Engineering in the University of Nottingham, Nottingham, UK. During April-July 2019, he was a Visiting PhD Student of the Department of Energy Technology in Aalborg University, Aalborg, Denmark. He was a Postgraduate Student in Department of Aircraft Design, Beihang University, Beijing, China from 2014 to 2017.

He serves as a Reviewer in the IEEE Transactions on Industrial Electronics and a session cochair in AIAA-EATS 2020. His research interests include filter design and optimization, model predictive control, search algorithms, machine learning applications on aircraft actuators.

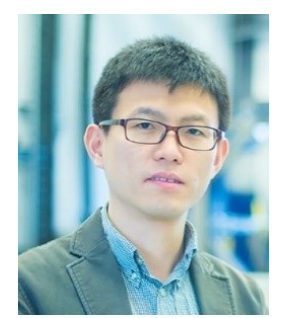

Tao Yang (M'16-SM'20) received his Ph.D. degree in electrical engineering from the University of Nottingham, Nottingham, UK, in 2013. Since then, he has been a Researcher, an Assistant Professor (2016) and is now an Associate Professor (2019) within the Power Electronics, Machines and Control Group, University of Nottingham.

His research expertise includes high-speed electric motor drive control, power electronic conversion, electrical system design and optimization for moreelectric/hybrid/all-electric aircraft applications.

Dr. Yang is an Associate Editor of IEEE Transactions on Transportation Electrification and Chinese Journal of Aeronautics.

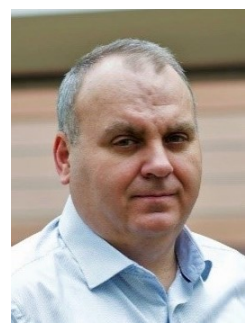

Serhiy Bozhko (M'96-SM'18) received the M.Sc. (Hons.) and Ph.D. degrees in electromechanical systems from the National Technical University of Ukraine, Kyiv, Ukraine, in 1987 and 1994, respectively. Since 2000, he has been with the Power Electronics, Machines and Control (PEMC) Research Group, University of Nottingham, Nottingham, U.K., where he is currently a Professor leading several Euand industry-funded projects in the area of aircraft electric power systems, including control, power quality and stability issues, power management and optimization, as well as advanced modeling and simulations methods.

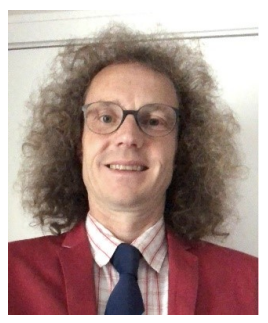

Pat Wheeler received his BEng [Hons] degree in 1990 from the University of Bristol, UK. He received his $\mathrm{PhD}$ degree in Electrical Engineering for his work on Matrix Converters from the University of Bristol, UK in 1994. In 1993 he moved to the University of Nottingham and worked as a research assistant in the Department of Electrical and Electronic Engineering. In 1996 he became a Lecturer in the Power Electronics, Machines and Control Group at the University of Nottingham, UK. Since January 2008 he has been a Full Professor in the same research group. He was Head of the Department of Electrical and Electronic Engineering at the University of Nottingham from 2015 to 2018.

$\mathrm{He}$ is currently the Head of the Power Electronics, Machines and Control Research Group, Global Director of the University of Nottingham's Institute for Aerospace Technology and is the Li Dak Sum Chair Professor in Electrical and Aerospace Engineering. He is a member of the IEEE PELs AdCom and was an IEEE PELs Distinguished Lecturer from 2013 to 2017. He has published 500 academic publications in leading international conferences and journals.

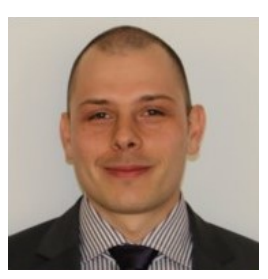

Tomislav Dragičević (S'09-M'13-SM'17) received the M.Sc. and the industrial Ph.D. degrees in Electrical Engineering from the Faculty of Electrical Engineering, University of Zagreb, Croatia, in 2009 and 2013, respectively. From 2013 until 2016 he has been a Postdoctoral researcher at Aalborg University, Denmark. From 2016 until 2020 he was an Associate Professor at Aalborg University, Denmark. From 2020 he is a Professor at the Technical University of Denmark.

$\mathrm{He}$ made a guest professor stay at University of Nottingham, UK during spring/summer of 2018. His research interest is application of advanced control, optimization and artificial intelligence inspired techniques to provide innovative and effective solutions to emerging challenges in design, control and cyber-security of power electronics intensive electrical distributions systems and microgrids. He has authored and coauthored more than 230 technical publications (more than 100 of them are published in international journals, mostly in IEEE), 8 book chapters and a book in the field.

He serves as an Associate Editor in the IEEE TRANSACTIONS ON INDUSTRIAL ELECTRONICS, in IEEE TRANSACTIONS ON POWER ELECTRONICS, in IEEE Emerging and Selected Topics in Power Electronics and in IEEE Industrial Electronics Magazine. Dr. Dragičević is a recipient of the Končar prize for the best industrial PhD thesis in Croatia, a Robert Mayer Energy Conservation award, and he is a winner of an Alexander von Humboldt fellowship for experienced researchers. 